Sonderheft 5 zu Band 26,

Oktober 2003

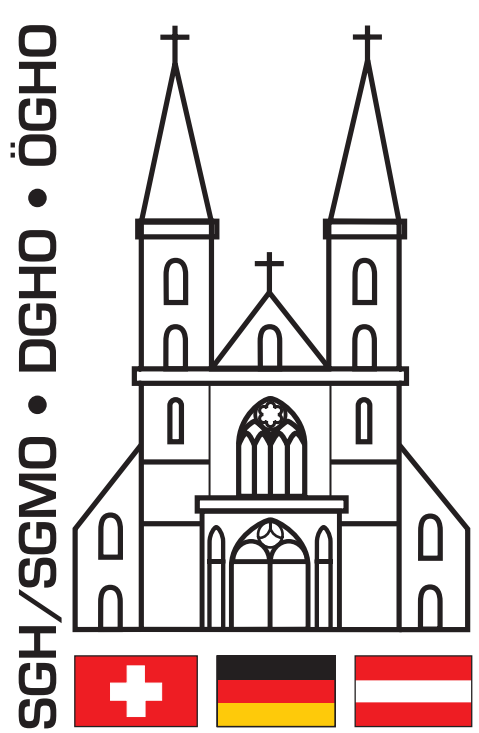

Gemeinsame Jahrestagung der Deutschen, Österreichischen und Schweizerischen Gesellschaften für Hämatologie und Onkologie

Basel, Schweiz, 4.-8. Oktober 2003

\title{
ABSTRACTS
}

Gast-Herausgeber

R. Herrmann, J. Passweg, C. Rochlitz, Basel, Schweiz 
Kongresspräsident

Wissenschaftliche Sekretäre

Lokales Organisationskomitee

Wissenschaftliches Komitee

Leitung Pflegetagung

Lokales Organisationskomitee Pflegetagung

Wissenschaftliches Sekretariat Pflegetagung

Abstractbegutachter
R. Herrmann

J. Passweg, C. Rochlitz

B. Biedermann, A. Gratwohl, L. Jost, A. Lohri, C. Ludwig, G. Marbet, R. Skoda, A. Tichelli

C. Aul, C. Benz, P. Beris, J. Beyer, C. Bokemeyer, C. Dittrich, J. Drach, M. Eilers, A. Filipowicz, C. Fonatsch, H. Gadner, A. Ganser, W. Gassmann, G. Gastl,

N. Gattermann, M. Ghielmini, U. Hess, W. Hiddemann, G. Holländer, D. Hölzer,

D. Huhn, B. Keppler, A. Kiss, U. Kleeberg, A. Knuth, C.-H. Köhne, T. Kroner,

S. Leyvraz, H. Ludwig, A. Mackensen, C. Manegold, G. Maschmeyer, J. Meran, T. Möröy, J. Nadig, A. Neubauer, N. Niederle, H. Ostermann, H. Pahl,

M. Pfreundschuh, M. Pless, J. Pont, K. Possinger, M. Primig, H. Riess,

C. Rüegg, A.-P. Sappino, H. Sauer, W. Scheithauer, L. Schmid,

H. Schrezenmeier, M. Schuler, R. Stahel, H.T. Steinmetz, D. Trump, L. Trümper, H. Veelken, E. Zucca

H. Stoll

P. Kammerer, S. Dinkler, H. Scheel, P. Jermann

M. Fliedner, C. Pino Molina, E. Haegi-Rieder

C. Aul, W. Berdel, P. Beris, H. Bernhard, J. Beyer, B. Biedermann, G. Bodoky,

C. Bokemeyer, M. Borner, A. Büchner, K. Buser, M. Castiglione, T. Cerny,

V. Diehl, P.-Y. Dietrich, C. Dittrich, B. Dörken, J. Drach, R. Dummer,

W. Eberhardt, S. Eychmüller, M. Fey, A. Filipowics, J. Finke, C. Fonatsch, M. Freund, A. Ganser, G. Gast, N. Gattermann, M. Ghielmini, S. Gillessen,

N. Gökbuget, H. Goldschmidt, A. Gratwohl, R. Haas, T. Haferlach,

R. Hartenstein, R. Hehlmann, E. Heidemann, V. Heinemann, R. Herrmann,

U. Hess, E. Hiller, A. Hochhaus, S. Hofer, E. Holler, D. Hölzer, D. Huhn, R. Issels,

E. Jäger, R. Joss, L. Jost, L. Kanz, U. Keilholz, B. Keppler, P. Ketterer,

U. Kleeberg, M. Kneba, A. Knuth, C.-H. Köhne, H.-J. Kolb, B. Lämmle, A. Lohri,

C. Ludwig, H. Ludwig, D. Lutz, A. Mackensen, C. Manegold, G. Marbet,

G. Maschmeyer, P. Miny, R. Morant, J. Nadig, A. Neubauer, N. Niederle,

D. Niederwieser, A. Ochsenbein, H. Ostermann, H. Pahl, J. Passweg,

C. Peschel, M. Pfreundschuh, G. Pichert, M. Pless, J. Pont, K. Possinger, M. Primig, P. Reichardt, P. Reusser, H. Riess, C. Rochlitz, A. Roth, C. Rüegg, M. Salzberg, A.-P. Sappino, H. Sauer, W. Scheithauer, P. Schmid, C. Schmidt, I. Schmidt-Wolf, N. Schmitz, H. Schmoll, H. Schrezenmeier, M. Schuler,

R. Skoda, H. Steinmetz, R. Stupp, E. Thiel, B. Thürlimann, A. Tichelli, A. Tobler,

L. Trümper, H. Veelken, M. Wernli, A. Wiestner, K. Wilms, A. Zander,

C. Zielinski, E. Zucca

M. Fliedner, B. Glawogger, E. Haegi-Rieder, C. Pino Molina, H. Stoll,

Young Masters Preis Jury

M. Fey, D. Huhn, D. Hossfeld, D. Lutz, A. Neubauer 


\section{Contents : Inhalt}

Onkologie 2003;26(Sonderheft 5)

Oral session:

Acute leukemia I

Urogenital tract cancer

V137 - V142

1

V143 - V148

V149 - V154

Lymphoma I

V180 - V185

Tumor immunology

Tumor pathophysiology

$\mathrm{V} 186-\mathrm{V} 191$

V192 - V197

Scientific symposium:

Sarcoma

203

12

Methodology in early clinical trials (CESAR)

Oral session:

Lymphoma II

$\mathrm{V} 213-\mathrm{V} 218$

13

Acute leukemia II

V219 - V224

16

Immunotherapy

$\mathrm{V} 225$ - V230

Hodgkin's lymphoma

$\mathrm{V} 260$ - V265

Breast cancer

V266 - V271

Hemostasis / Anemia

V272 - V277

Lung cancer

V304 - V309

26

Plasma cell dyscrasia

V310 - V315

28

Infections

V316 - V321

30

Gastrointestinal cancer

V338 - V343

32

Hematopoietic stem cell transplantation I

V344 - V349

34

CNS / Head and neck tumors

V350 - V355

36

Hematopoietic stem cell transplantation II

V372-V378

38

Melanoma / Sarcoma

V379-V384

40

Genomics / Proteomics / Gene therapy

V385 - V391

Scientific symposium:

MPS 
Oral session:

Colorectal cancer

$\mathrm{V} 403-\mathrm{V} 412$

MDS / MPS

\section{Poster session:}

Genomics / Proteomics / Gene therapy

$\mathrm{P} 429-\mathrm{P} 455$

Hemostasis / Anemia

P456-P475

Hematopoietic stem cell transplantation

P476 - P547

63

Leukemia

P548 - V633

83

MDS / MPS

Plasma cell dyscrasia

Stem cell biology

$\mathrm{P} 634-\mathrm{P} 683$

Lung cancer

$\mathrm{P} 684$ - P722

P723 - P751

Gastrointestinal cancer

P753 - P767

Infections / Supportive care

$\mathrm{P} 768$ - P797

144

Lymphoma

$\mathrm{P} 798-\mathrm{P} 820$

153

P821 - P911

160

Hodgkin's Iymphoma

P912 - P919

185

Breast cancer

New drugs

Melanoma / Sarcoma

$\mathrm{P} 920$ - P932

187

P933 - P946

Urogenital tract cancer

P947 - P960

CNS / Head and neck tumors

P961 - P974

Tumor immunology / Immunotherapy

$\mathrm{P} 975$ - P984

202

Tumor pathophysiology

P985 - P1043

204

P1044 - P1070

220

\section{Pflegetagung:}

Qualitätssicherung

$1073-1076$

227

Symptomkontrolle

$1078-1084$

229

Schmerzlinderung

$1087-1090$

231

Palliation - Patienten und Angehörige

$1091-1097$

232

Palliation - Institutionen

1100

235

Author Index

Imprint

Guidelines for Authors 